\title{
Breaking the Silence: Recognizing Sexual Violence in Criminal Justice Reform
}

\author{
Michele R. Decker and Susan G. Sherman
}

There is unprecedented attention in the USA on police violence against the communities in which they are meant to protect and serve. For many Americans, Ferguson, MO, and Baltimore, MD, have brought to the fore what many citizens-primarily African American males-have experienced regularly for decades. The deaths of Michael Brown, Freddie Grey, and countless others have generated extensive public and criminal justice dialogue about harmful police practices and the systems that enable them. These homicides, rare and egregious, represent the tip of the proverbial iceberg of police relations that undermine citizen safety and have thus catalyzed calls for reform.

Women's health and well-being are undermined by a parallel reality of inaction, mistreatment, and abuse perpetrated by individuals in positions of power, predominantly in the realm of sexual harassment and violence. The lack of "bodies" as evidence of such exploitation of power renders these acts effectively invisible to the public eye and therefore policy discourse.

This mistreatment is exemplified by the systematic mishandling and dismissal of rape cases, ${ }^{1,2}$ sexual abuse in detention centers, ${ }^{3}$ coerced sex in exchange for routine repairs in public housing in Baltimore, $\mathrm{MD}$, and in a recent extreme case in Oklahoma City, OK, the targeted rape of African-American women at the hands of a single white policeman, Daniel Holtzclaw. Law enforcement abuses against women are enabled by the same power imbalances and systemic racial and economic biases that give rise to young black men's mistreatment, including death, at the hands of police. Inaction, mistreatment, and abuse against women are further fueled by gender-based power imbalances and the age-old threats of sexual violence and sexual shaming.

Yet this reality remains largely cloaked in silence. Why? Too many sexual violence victims are reluctant to report to police or media for shame, blame, and fear of retribution and a sense of futility in the justice system-all fears exploited by perpetrators to garner silence and compliance. The secluded locations in which sexual violence often occurs are out of sight, further contributing to a culture of silence. When perpetrators themselves are agents of the state, an added power imbalance emerges in asking law enforcement agents to hold one of their own accountable. For women involved in drugs, sex trade, or other criminal activity, as intentionally targeted by Daniel Holtzclaw, the power imbalances are ever sharper

Decker is with the Department of Population, Family \& Reproductive Health, Johns Hopkins Bloomberg School of Public Health, Baltimore, MD, USA; Decker and Sherman are with the Women's Health \& Rights Program, Center for Public Health and Human Rights, Johns Hopkins Bloomberg School of Public Health, Baltimore, MD, USA; Sherman is with the Department of Health, Behavior \& Society, Johns Hopkins Bloomberg School of Public Health, Baltimore, MD, USA.

Correspondence: Michele R. Decker, Women's Health \& Rights Program, Center for Public Health and Human Rights, Johns Hopkins Bloomberg School of Public Health, Baltimore, MD, USA. (E-mail: mdecker@jhu.edu) 
and barriers to reporting ever greater. Our own local and global research reveals these forces in enabling violence and harassment with impunity among women involved in sex work and drugs. ${ }^{4-7}$

Often, the very nature of where and who is targeted reveals the exploitation of intersecting power imbalances along lines of race, gender, social status, and criminal involvement. The Holtzclaw example in particular demonstrates the selection of individuals and locations that effectively provide "cover" and the expectation of impunity. In short, the perpetrators assumed that none of these women stood a chance of being heard-an assumption that proved mostly correct.

What is the solution? The charges against Holtzclaw were catalyzed by one woman who defied the odds. We cannot risk women's lives and safety on the courage of single individuals. Rather, our policies and practices must assure victims access, equity, and safety in the justice process and actively foster it. Ensuring that criminal justice reforms address sexual violence requires understanding and mitigating the dynamics that can intersect to undermine justice and risk exploitation, including race, gender, drugs, sex work, and criminal history. These factors must be attended to in policy, practice, and the data with which to evaluate progress. Not all law enforcement agents are abusive, and no justice is served in pitting sexual violence against other forms of mistreatment. Public safety and public health are not advanced by a witch-hunt to weed out the bad guys, but rather through supporting systems that enable transparency, vigilant monitoring, accountability, and action.

Recent and longstanding tools can be leveraged to achieve these goals. The recent release of the US Department of Justice Guidance on Gender Bias (Identifying and Preventing Gender Bias in Law Enforcement Response to Sexual Assault and Domestic Violence, 2015) calls on law enforcement agencies to incorporate a set of principles into clear policies, training, and supervision protocols; among them include the accountability of officers who commit sexual assault or domestic violence, and maintaining, reviewing, and acting on data on sexual assault and domestic violence. For the implementation of this Guidance, we argue for documentation, clear mechanisms, and response provisions to enable justice for women with a criminal history, and those involved in drugs and sex work. Documentation systems for reported police sexual abuse and misconduct are essential to monitor abuses against both men and women, as are systems for investigation and holding perpetrators accountable. The implementation of these systems must be monitored to ensure they are accessible, meaningful, and effective. Given the many barriers to reporting, mechanisms must exist for victims to feel comfortable and safe bringing cases of violence forward. The comprehensive federal Violence Against Women Act of 1994 blends provisions for perpetrator accountability with support for victims of violence. Criminally involved women have not historically been enumerated among the underserved populations addressed by VAWA, though this population would benefit from targeted outreach by rape crisis and domestic violence programs to ensure their access to support and justice. The National Intimate Partner and Sexual Violence Survey (NISVS) could monitor trends in prevalence and access to justice by including elements on criminal history, discrimination, and fear in accessing justice. The 2011-2012 Progress of the World's Women report on access to justice ${ }^{8}$ provides direction for systems reform.

We must harness these tools to ensure inclusion of sexual violence in justice reform and bring to fore the prevalence, severity, and risks of inaction regarding sexual assault in its many manifestations. The next steps are challenging in that they will take strong leadership and discomfort in changing the prevailing norms and 
practices. Sexual violence against women-whether systematic rape, "benign" forms of sexual harassment, or inaction in response, must be part of the public discourse and police reform. Gender-based violence affects an estimated one in three women globally with significant implications for morbidity and mortality. ${ }^{9}$ Without addressing violence against women, our public dialogue on police reform will fall short, and women's health and safety will continue to suffer.

\section{ACKNOWLEDGMENTS}

Authors' contributions. MRD and SGS conceptualized the commentary. MRD led the writing, and SGS provided critical review and input. All authors reviewed and approved the commentary prior to submission.

\section{COMPLIANCE WITH ETHICAL STANDARDS}

Ethics committee approval. Not applicable.

\section{REFERENCES}

1. Fenton J. City rape statistics, investigations draw concern. Baltimore Sun. 2010 June 27.

2. Human Rights Watch. Capitol Offense: police mishandling of sexual assault cases in the District of Columbia. New York, NY: Human Rights Watch; 2013.

3. Weiser B. 2 Rikers inmates accuse guard of repeated rapes. New York Times. 2015 May 20: A27.

4. Lim S, Peitzmeier S, Cange C, et al. Violence against female sex workers in Cameroon: accounts of violence, harm reduction, and potential solutions. J Acquir Immune Defic Syndr. 2015; 68(Suppl 2): S241-S247.

5. Sherman SG, Footer K, Illangasekare S, Clark E, Pearson E, Decker MR. "What makes you think you have special privileges because you are a police officer?" A qualitative exploration of police's role in the risk environment of female sex workers. AIDS Care. 2015; 27(4): 473-480.

6. Decker MR, Pearson E, Illangasekare SL, Clark E, Sherman SG. Violence against women in sex work and HIV risk implications differ qualitatively by perpetrator. BMC Public Health. 2013; 13: 876.

7. Decker MR, Crago AL, Chu SK, et al. Human rights violations against sex workers: burden and effect on HIV. Lancet. 2015; 385(9963): 186-199.

8. UNWomen. 2011-2012 Progress of the World's Women: In Pursuit of Justice. New York, NY: UNWomen; 2011.

9. World Health Organization. Global and regional estimates of violence against women: prevalence and health effects of intimate partner violence and non-partner sexual violence. Geneva, Switzerland: WHO; 2013. 